\title{
ON RIEMANNIAN CURVATURE OF HOMOGENEOUS SPACES
}

\section{SIGURĐUR HELGASON}

1. Introduction. When developing his theory of symmetric spaces, E. Cartan proved that a compact symmetric Riemannian space has sectional curvature everywhere $\geqq 0$ and that a noncompact irreducible symmetric Riemannian space has sectional curvature everywhere $\leqq 0$. H. Samelson has recently [5] proved an analogue of Cartan's theorem for the compact case, namely that a homogeneous space $G / K$ where $G$ is a connected compact Lie group, $K$ a closed subgroup, has sectional curvature everywhere $\geqq 0$. Here the metric on $G / K$ is the one that is obtained from a two-sided invariant metric on $G$ by the natural projection. While Samelson's proof is simple and geometric it gives no information in the noncompact case.

In the present paper we give a proof of the theorem of Samelson by a method which furnishes some additional information and which can be used to prove Cartan's theorem for the noncompact case as well as for the compact case.

2. Preliminaries. Let $G$ be a connected Lie group with Lie algebra g. Let $g \rightarrow \operatorname{Ad}(g)$ denote the adjoint representation of $G$ on $g$ and let $X \rightarrow \operatorname{ad}(X)$ denote the adjoint representation of $\mathfrak{g}$ on $\mathfrak{g}$ so $\operatorname{ad}(X)(Y)$ $=[X, Y]$. Suppose $K$ is a closed subgroup of $G$ such that $\operatorname{Ad}_{G}(K)$ (the image of $K$ under $g \rightarrow \operatorname{Ad}(g)$ ) is compact. If the Lie algebra of $K$ is $\mathfrak{l}$ there exists a subspace $\mathfrak{m} \subset \mathfrak{g}$ such that $\mathfrak{g}=\mathfrak{m}+\mathfrak{f}$ (direct sum of vector spaces) and such that $\operatorname{Ad}_{G}(k) \mathfrak{m} \subset \mathfrak{m}$ for all $k \in K$. The manifold $G / K$ of left cosets $g K$ can be given the structure of a Riemannian manifold whose metric is invariant under $G$, that is for each $x \in G$ the mapping $\tau(x): g K \rightarrow x g K$ of $G / K$ onto $G / K$ is an isometry. The natural projection $\pi$ of $G$ onto $G / K$ maps $m$ isomorphically onto the tangent space to $G / K$ at $\pi(e)$ ( $e$ is the identity element of $G$ ) in such a way that the action of $\operatorname{Ad}_{G}(K)$ on $\mathrm{m}$ corresponds to the action of $\tau(K)$ on the tangent space. Thus a Riemannian metric on $G / K$ invariant under all $\tau(x), x \in G$ is uniquely determined by a positive definite quadratic form on $\mathrm{m}$, invariant under $\operatorname{Ad}_{G}(K)$. The space $G / K$ is called a symmetric Riemannian homogeneous space if the subspace $m$ above satisfies $[\mathfrak{m}, \mathfrak{m}] \subset \mathfrak{f}$. For such a space we have then the relations

$$
\mathfrak{g}=\mathfrak{m}+\mathfrak{t},[\mathfrak{m}, \mathfrak{m}] \subset \mathfrak{f},[\mathfrak{m}, \mathfrak{f}] \subset \mathfrak{m},[\mathfrak{f}, \mathfrak{f}] \subset \mathfrak{f} .
$$

Received by the editors May 23, 1958. 
The space $G / K$ will be called irreducible if $\operatorname{Ad}_{G}(K)$ acts irreducibly on $\mathrm{m}$.

3. The exponential mapping of a symmetric space. Let $G / K$ be a symmetric Riemannian space. The subspace $\mathfrak{m}$ of $\mathfrak{g}$ can be identified with the tangent space to the complete Riemannian manifold $G / K$ at $\pi(e)$. Let Exp denote the mapping of $m$ into $G / K$ which maps straight lines through 0 in $\mathfrak{m}$ onto geodesics through $\pi(e)$ in $G / K$, preserving lengths of segments of each such line.

An important theorem in the theory of symmetric spaces states that each geodesic through $\pi(e)$ is an orbit of a one-parameter group of "transvections" (see $[1 ; 4]$ ) which can be expressed

$$
\operatorname{Exp} X=\pi \circ \exp X \quad \text { for } X \in \mathfrak{m} \text {. }
$$

For each $X \in \mathfrak{m}$, let $T_{X}$ denote the restriction of $(\operatorname{ad} X)^{2}$ to $\mathfrak{m}$. From the relations (1) we see that $T_{X}$ maps $\mathfrak{m}$ into itself. We consider $\mathfrak{m}$ as a manifold whose tangent space at each point is identified with $\mathfrak{m}$ itself under the usual identification of parallel vectors.

TheOREM 1. The differential of the mapping Exp satisfies

$$
d \operatorname{Exp}_{X}=d \tau(\exp X) \circ \sum_{n=0}^{\infty} \frac{T_{X}^{n}}{(2 n+1) !} \quad \text { for } X \in \mathfrak{m} .
$$

This theorem is useful because it describes the Exp-mapping by means of an isometry and a linear transformation of $\mathfrak{m}$ which is given in terms of the Lie algebra.

We first prove a lemma which describes the analogous situation on $G$. This lemma is essentially equivalent to Cartan's formula which expresses the Maurer-Cartan forms in canonical cordinates and is proved in [3, p. 157]. We give a different proof here.

For each $h \in G$, let $L(h)$ denote the left translation $g \rightarrow h g$ on $G$.

Lemma 2. Let $G$ be a connected Lie group with Lie algebra g. Identifying $\mathfrak{g}$ with its tangent space at each point we have

$$
d \exp _{X}=d L(\exp X) \circ \frac{1-e^{-\operatorname{ad} X}}{\operatorname{ad} X} \quad \text { for } X \in \mathfrak{g} .
$$

Proof. If $D$ is a linear operator on the space $C^{\infty}(G)$ of indefinitely differentiable functions on $G$ and $F \in C^{\infty}(G),[D F](g)$ will denote the value of $D F$ at $g$. Each $Z \in \mathrm{g}$ gives rise to a left invariant vector field on $G$ and therefore to an operator $F \rightarrow Z F$ on $C^{\infty}(G)$ which commutes with left translations on $G$. The value of the function $Z F$ at $g$ is given by 


$$
[Z F](g)=\lim _{t \rightarrow 0} \frac{F(g \exp t Z)-F(g)}{t} .
$$

It follows by a simple induction that $d^{n} / d u^{n} F(\exp u Z)=\left[Z^{n} F\right](\exp u Z)$ which by Taylor's formula implies

$$
f(g \exp t Z)=\sum_{n=0}^{\infty} \frac{t^{n}}{n !}\left[Z^{n} f\right](g)
$$

if $f$ is analytic in a neighborhood of $g$ and $t$ is sufficiently small. Also the Lie algebra element $[X, Y]$ induces in the same fashion the operator $X Y-Y X$. Now suppose $f$ is analytic in a neighborhood $V$ of $e$ in $G$ and that $U$ is an open neighborhood of 0 in $g$ such that $\exp U \subset V$. Let $X \in U$. Each $Y \in \mathfrak{g}$ gives by parallel translation a tangent vector to $\mathfrak{g}$ at $X$ and

$$
\begin{aligned}
{\left[d \exp _{X}(Y) f\right](\exp X) } & =[Y(f \circ \exp )](X)=\left[\frac{d}{d t} f(\exp (X+t Y))\right] \\
& =\frac{d}{d t}\left\{\sum_{0}^{\infty} \frac{1}{n !}\left[(X+t Y)^{n} f\right](e)\right\}_{t=0} .
\end{aligned}
$$

Due to the analyticity of $f$ the growth of its derivatives is so restricted that the series in the last expression above can be differentiated with respect to $t$, term by term. Only the first power of $t$ gives a contribution so we obtain

$$
\begin{aligned}
& {\left[d \exp _{X}(Y) f\right](\exp X)} \\
& \quad=\sum_{n=0}^{\infty} \frac{1}{(n+1) !}\left[\left(Y X^{n}+X Y X^{n-1}+\cdots+X^{n} Y\right) f\right](e) .
\end{aligned}
$$

To simplify this last expression we use the formula

$$
Y X^{m}=\sum_{p=0}^{m}(-1)^{p} C_{m, p} X^{m-p}(\operatorname{ad} X)^{p}(Y) \quad X, Y \in \mathfrak{g} .
$$

For $m=1$ this amounts to the definition of ad $X$ and for a general integer $m>0$ it follows easily by induction. Using the relation $\sum_{p=0}^{n-k} C_{n-p, k}=C_{n+1, k+1}$ we obtain

$$
\begin{aligned}
Y X^{n}+X Y X^{n-1}+\cdots+ & X^{n} Y \\
& =\sum_{p=0}^{n} X^{p} \sum_{k=0}^{n-p}(-1)^{k} C_{n-p, k} X^{n-p-k}(\operatorname{ad} X)^{k}(Y) \\
& =\sum_{k=0}^{n} C_{n+1, k+1}(-1)^{k} X^{n-k}(\operatorname{ad} X)^{k}(Y)
\end{aligned}
$$


which combined with (4) and (5) yields

$$
\begin{aligned}
{\left[d \exp _{X}(Y) f\right](\exp X) } & =\sum_{r=0}^{\infty}\left[\frac{X^{r}}{r !} \sum_{m=0}^{\infty}(-1)^{m} \frac{1}{(m+1) !}(\operatorname{ad} X)^{m}(Y) f\right](e) \\
& =\left[\frac{1-e^{-\operatorname{ad} X}}{\operatorname{ad} X}(Y) f\right](\exp X) .
\end{aligned}
$$

This proves the lemma for all $X \in U$. Its validity for all of $\mathfrak{g}$ is obtained by analytic continuation as follows: Let $Y_{1}, \cdots, Y_{n}$ be a basis of $\mathfrak{g}$ and for each $X \in \mathfrak{g}$ put $Y_{i}^{*}=d L(\exp X)\left(Y_{i}\right)$; then $Y_{i}^{*}$, $i=1,2, \cdots, n$ is a basis for the tangent space to $G$ at $\exp X$. Define the functions $t_{i j}(X)$ by $d \exp _{X}\left(Y_{i}\right)=\sum_{j} t_{i j}(X) Y_{j}^{*}$. Then each $t_{i j}(X)$ is an analytic function on $\mathrm{g}$. On the other hand

$$
\frac{1-e^{-\operatorname{ad} X}}{\operatorname{ad} X}\left(Y_{i}\right)=\sum_{j} s_{i j}(X) Y_{j}
$$

where $s_{i j}(X)$ are analytic functions on $\mathfrak{g}$. We have proved that $t_{i j}(X)=s_{i j}(X)$ for all $X \in U$ and all $i, j$. But since $t_{i j}$ and $s_{i j}$ are analytic functions on $\mathfrak{g}$ this last equation holds for all $X$ and the lemma follows.

Theorem 1 now follows easily. From the relation $\pi \circ L(g)$ $=\tau(g) \circ \pi$ and (2) we obtain for $Y \in \mathfrak{m}$

$$
\begin{aligned}
d \operatorname{Exp}_{X}(Y) & =d \pi \circ d \exp _{X}(Y)=d \pi \circ d L(\exp X) \circ \frac{1-e^{-\mathrm{ad} X}}{\operatorname{ad} X}(Y) \\
& =d \tau(\exp X) \circ d \pi \sum_{0}^{\infty}(-1)^{m} \frac{(\operatorname{ad} X)^{m}}{(m+1) !}(Y)
\end{aligned}
$$

From the relations (1) it follows that

$$
d \pi \circ(\operatorname{ad} X)^{m}(Y)=\left\{\begin{array}{cl}
\left(T_{X}\right)^{n} & \text { if } m=2 n, \\
0 & \text { if } m \text { is odd }
\end{array}\right.
$$

which proves Theorem 1.

4. The Riemannian curvature of a symmetric space. Let $M$ be a Riemannian manifold, $m$ a point in $M, M_{m}$ the tangent space to $M$ at $m$. The mapping $\operatorname{Exp}_{m}$ maps a neighborhood of 0 in $M_{m}$ onto a neighborhood of $m$ in $M$ in a one-to-one fashion such that line segments through 0 go into geodesics through $m$ in $M$. Let $S$ be a twodimensional subspace of $M_{m}$ and $K(S)$ the corresponding sectional curvature. In order to apply Theorem 1 it is convenient to derive a new expression for $K(S)$. 
Lemma 3. Let $\Delta$ denote the Laplacian of the metric vector space $S$ above and let $f$ be the Radon-Nikodym derivative of the restriction of $\operatorname{Exp}_{m}$ to $S$ (the ratio of the volume elements in $\operatorname{Exp}_{m}(S)$ and $S$ ) normalized by $f(0)=1$. Then

$$
K(S)=-\frac{3}{2} \Delta f(0) .
$$

Proof. Let $A_{0}$ denote a small disk in $S$ with center at 0 and radius $r$ and we put $A=\operatorname{Exp}_{m}\left(A_{0}\right)$. Let $A_{0}(r)$ and $A(r)$ denote the corresponding areas. Then

$$
A(r)=\int_{A_{0}} f(X) d X=A_{0}(r)\left\{f(0)+\frac{1}{8} r^{2}[\Delta f](0)+\cdots\right\} .
$$

Applying Vermeil's formula

$$
K(S)=\lim _{r \rightarrow 0} 12 \frac{A_{0}(r)-A(r)}{r^{2} A_{0}(r)},
$$

[2, p. 253], we get Lemma 3 immediately.

Theorem 2. Let $G / K$ be a symmetric Riemannian space, $Q$ the quadratic form on $\mathfrak{m}$ that gives the invariant metric on $G / K$. Let $S$ be a two-dimensional subspace of $\mathfrak{m}$ spanned by the orthonormal vectors $Y$ and $Z$. Then

$$
K(S)=-Q\left(T_{Y}(Z), Z\right) .
$$

Proof. Let $X_{1}, \cdots, X_{n}$ be a basis of $\mathfrak{m}$ such that $Q\left(X_{i}, X_{j}\right)=\delta_{i}{ }^{j}$ and such that $X_{1}=Y, X_{2}=Z$. Each $X \in S$ can be represented $X$ $=x_{1} X_{1}+x_{2} X_{2}$ and the Laplacian $\Delta$ on $S$ has the form

$$
\Delta=\frac{\partial^{2}}{\partial x_{1}^{2}}+\frac{\partial^{2}}{\partial x_{2}^{2}} .
$$

If $a$ and $b$ are two vectors in a metric vector space we denote by $a \bigvee b$ the parallelogram spanned by $a$ and $b$ and by $|a \bigvee b|$ the area. We write $A_{X}=\sum_{0}^{\infty}[(2 n+1) !]^{-1} T_{X}^{n}$ and $A_{X}\left(X_{i}\right)=v_{i}, i=1$, 2 . Since the mapping $\tau(\exp X)$ is an isometry we see from Theorem 1

$$
f(X)=\frac{\left|v_{1} \vee v_{2}\right|}{\left|X_{1} \vee X_{2}\right|}=\left|v_{1} \vee v_{2}\right| .
$$

If $A_{X}$ has the matrix $\left(A_{\imath j}\right)$ with respect to the basis $X_{1}, \cdots, X_{n}$,

$$
\begin{aligned}
f(X) & =\left|\left(A_{11} X_{1}+\cdots+A_{n 1} X_{n}\right) \vee\left(A_{12} X_{1}+\cdots+A_{n 2} X_{n}\right)\right| \\
& =\left[\left(A_{11} A_{22}-A_{12} A_{21}\right)^{2}+\cdots+\left(A_{i 1} A_{j 2}-A_{j 1} A_{i 2}\right)^{2}+\cdots\right]^{1 / 2}
\end{aligned}
$$


since $\left|\left(A_{i 1} A_{j 2}-A_{j 1} A_{i 2}\right)\right|$ is the area of the projection of $v_{1} \bigvee v_{2}$ on the $\left(X_{i}, X_{j}\right)$-plane. In computing $\Delta f(0)$ from the expression for $f(X)$ we only have to consider terms of second order in $x_{1}$ and $x_{2}$. Since the matrix elements $T_{i j}$ of $T_{X}$ are either 0 or are of second order in $x_{1}$ and $x_{2}$ we find easily

$$
\begin{aligned}
{[\Delta f](0) } & =\left[\Delta A_{11} A_{22}\right](0)=\left[\Delta\left(1+\frac{1}{3 !} T_{11}\right)\left(1+\frac{1}{3 !} T_{22}\right)\right] \\
& =\frac{1}{3 !}\left[\Delta\left(T_{11}+T_{22}\right)\right](0)
\end{aligned}
$$

and since $T_{X}=\left(\operatorname{ad}\left(x_{1} X_{1}+x_{2} X_{2}\right)\right)^{2}$ restricted to $\mathfrak{m}$,

$$
\begin{aligned}
{[\Delta f](0)=\frac{1}{3}\left[Q\left(T_{Y}(Z), Z\right)+Q\left(T_{Z}(Y), Y\right)+Q\left(T_{Y}(Y), Y\right)\right.} & \\
& \left.+Q\left(T_{Z}(Z), Z\right)\right] .
\end{aligned}
$$

Here the last two terms vanish, the two first are equal and the theorem follows from Lemma 3.

Theorem 3. Let $G / K$ be an irreducible symmetric Riemannian space.

(i) If $G / K$ is compact the sectional curvature is everywhere $\geqq 0$.

(ii) If $G / K$ is noncompact the sectional curvature is everywhere $\leqq 0$.

Proof. We can assume that $G$ acts effectively on $G / K$; it is well known $[4, p .56]$ that either $\mathfrak{g}$ is semi-simple or $[\mathfrak{m}, \mathfrak{m}]=0$. In the latter case Theorem 3 is obvious so we assume $\mathfrak{g}$ is semi-simple. Let $B$ denote the Killing form on $g$. By the irreducibility

$$
B(X, X)=\lambda Q(X, X) \quad \text { for all } X \in \mathfrak{m}
$$

where $\lambda$ is a constant. Let $D$ be a positive definite quadratic form on $\mathfrak{f}$ invariant under $\operatorname{Ad}_{G}(K)$. The form $\Phi(Z, Z)=Q(X, X)+D(Y, Y)$ $(Z=X+Y, X \in \mathfrak{m}, Y \in \mathfrak{f})$ is positive definite and invariant under $\operatorname{Ad}_{G}(K)$; hence if $Y \in \mathfrak{l}$, the linear transformation ad $Y$ has a skew symmetric matrix with respect to $\Phi$ and

$$
B(Y, Y)=\operatorname{Tr}(\operatorname{ad} Y \operatorname{ad} Y) \leqq 0 .
$$

Now let $Z$ denote the center of $G$. Then the compact group $\operatorname{Ad}_{G}(K)$ is isomorphic to $K /(Z \cap K)$. But $Z \cap K=e$ since $G$ acts effectively so $K$ is compact. The constant $\lambda$ is negative in the case (i) and positive in case (ii). Using the notation of Theorem 2 we obtain from (8) $B\left(T_{Y}(Z), Z\right)=-B([Y, Z],[Y, Z]) \geqq 0$. Theorem 3 now follows from (7).

Remark. The conclusion of (i) in Theorem 3 holds whether or not 
$G / K$ is irreducible. This can be seen by decomposing $\mathfrak{m}$ into irreducible subspaces invariant under $\operatorname{Ad}_{G}(K)$ and orthogonal with respect to $Q$. On each of those subspaces $B$ is a nonpositive multiple of $Q$ and we can proceed as before.

5. Compact homogeneous spaces. The theorem of H. Samelson mentioned in the introduction can be stated as follows.

Theorem 4. Let $G$ be a compact connected Lie group, $K$ a closed subgroup. Let $Q$ be a positive definite quadratic form on the Lie algebra $\mathfrak{g}$ invariant under $\operatorname{Ad}(G)$ and let $\mathrm{m}$ be the orthogonal complement to the subalgebra $\mathfrak{f}$. The restriction of $Q$ to $\mathfrak{m}$ defines a Riemannian metric on $G / K$ invariant under $G$, and with respect to this metric the sectional curvature is everywhere $\geqq 0$.

Proof. In the two-sided invariant metric on $G$ given by $Q$ the geodesics are the cosets of one-parameter subgroups. This is a special case of (2) applied to the symmetric space $G=(G \times G) / D$ where $D$ is the diagonal of $G \times G$, the symmetry automorphism of $G \times G$ being $(x, y) \rightarrow(y, x)$ and each $\left(g, g_{1}\right) \in G \times G$ giving the isometry $x \rightarrow g x g_{1}^{-1}$ of $G$. The geodesics in $G / K$ through $\pi(e)$ are again projections of certain one-parameter subgroups in $G$, that is

$$
\operatorname{Exp} X=\pi \circ \exp X \quad \text { for } X \in \mathfrak{m} \text {. }
$$

(See [4, Theorem 13.2]. In [5] a simple geometric proof was based on comparison between lengths of curves in $G$ and their projections in $G / K$.) Now let $S$ be a two-dimensional subspace of $\mathfrak{m}$. We can then find an orthonormal base $\left(X_{i}\right)$ of $\mathfrak{m}$ and an orthonormal base $\left(X_{\alpha}\right)$ of such that $X_{1}$ and $X_{2}$ span $S$. Each $X \in S$ can be written $X=x_{1} X_{1}+x_{2} X_{2}$ and the Laplacian on $S$ is

$$
\Delta=\frac{\partial^{2}}{\partial x_{1}^{2}}+\frac{\partial^{2}}{\partial x_{2}^{2}} .
$$

We also put $\left[X_{i}, X_{j}\right]=\sum_{k} c_{i j k} X_{k}+\sum_{\rho} c_{i j \rho} X_{\rho}$ and $A_{X}=(\operatorname{ad} X)^{-1}$ $\cdot\left(1-e^{-\mathbf{a d} X}\right)$. Let $f$ and $F$ denote the Radon-Nikodym derivative of the restrictions of $\exp$ and Exp to $S$. Then

$$
f(X)=\frac{\left|A_{X}\left(X_{1}\right) \vee A_{X}\left(X_{2}\right)\right|}{\left|X_{1} \vee X_{2}\right|}
$$

and

$$
F(X)=\frac{\left|d \pi \cdot A_{X}\left(X_{1}\right) \bigvee d \pi \cdot A_{X}\left(X_{2}\right)\right|}{\left|X_{1} \bigvee X_{2}\right|}
$$


Now it is easily seen, that on cancelling terms of order $\geqq 2$ in $x_{1}$ and $x_{2}$ $A_{X}\left(X_{1}\right)=\left(1+p_{1}\right) X_{1}+p_{2} X_{2}+\cdots+p_{n} X_{n}+\cdots+p_{\rho} X_{\rho}+\cdots$, $A_{X}\left(X_{2}\right)=q_{1} X_{1}+\left(1+q_{2}\right) X_{2}+\cdots+q_{n} X_{n}+\cdots+q_{\sigma} X_{\sigma}+\cdots$

where the coefficients $p$ and $q$ are polynomials in $x_{1}$ and $x_{2}$ of degree $\geqq 1$ without constant terms. Moreover $p_{\rho}=-c_{21 \rho} x_{2} / 2, q_{\sigma}=-c_{12 \sigma} x_{\sigma} / 2$. The expressions for $d \pi \cdot A_{X}\left(X_{1}\right)$ and $d \pi A_{X}\left(X_{2}\right)$ are obtained by cancelling the $X_{\alpha}$ from the expressions above. By a computation similar to the one used in the proof of Theorem 2 we find

$$
\Delta f(0)=\Delta F(0)+\frac{1}{2} \sum_{\rho} c_{12 \rho}^{2} .
$$

By the remark following Theorem $3(G \times G) / D$ has curvature everywhere $\geqq 0$; by Lemma $3 \Delta f \leqq 0$ and by (10) $\Delta F \leqq 0$. This proves Theorem 4.

REMARK. From relation (10) it is easily seen that in the situation described in Theorem 4 , the curvatures of $G$ and $G / K$ are the same for all sections of $\mathfrak{m}$ if and only if $c_{i j p} \equiv 0$, that is $\mathfrak{m}$ is a subalgebra, hence an ideal in $\mathfrak{g}$.

\section{BiBLIOGRAPHY}

1. E. Cartan, La théorie des groupes finis et continus et l'analysis situs, Mémor. Sci. Math. fasc. XLII, Paris, 1930.

2. - Leģons sur la géometrie des espaces de Riemann, 2d ed., Paris, 1951.

3. C. Chevalley, Theory of Lie groups, Princeton, 1946.

4. K. Nomizu, Invariant affine connections on homogeneous spaces, Amer. J. Math. vol. 76 (1954) pp. 33-65.

5. H. Samelson, On curvature and characteristic of homogeneous spaces, Mich. Math. J. vol. 5 (1958) pp. 13-18.

University of Chicago 